\title{
Naringenin pre-treatment inhibits neuroapoptosis and ameliorates cognitive impairment in rats exposed to isoflurane anesthesia by regulating the PI3/Akt/PTEN signalling pathway and suppressing NF-kB-mediated inflammation
}

\author{
FU-ZHOU HUA $^{1 *}$, JUN YING ${ }^{1 *}$, JING ZHANG $^{1}$, XI-FENG WANG ${ }^{2}$, \\ YAN-HUI HU ${ }^{1}$, YING-PING LIANG ${ }^{1}$, QIN LIU $^{1}$ and GUO-HAI XU ${ }^{1}$ \\ ${ }^{1}$ Department of Anesthesiology, The Second Affiliated Hospital of Nanchang University, Nanchang, Jiangxi 330006;
${ }^{2}$ Department of Anesthesiology, The First Affiliated Hospital of Nanchang University, Nanchang, Jiangxi 330039, P.R. China
}

Received October 6, 2015; Accepted July 21, 2016

DOI: $10.3892 /$ ijmm.2016.2715

\begin{abstract}
The volatile anaesthetic isoflurane is one of the most frequently employed general anaesthetics in neonates, children and adults. Accumulating evidence demonstrated that exposure to anaesthetics is associated with widespread neurodegeneration and cognitive impairment. Thus, the identification and development of compounds capable of preventing or reducing these adverse effects is of great clinical importance. For this purpose, the present study aimed to assess the effects of a flavonoid, naringenin, on isoflurane-induced neuroapoptosis and cognitive impairment. Separate groups of neonatal rat pups were administered naringenin at 25,50 or $100 \mathrm{mg} / \mathrm{kg}$ body weight from postnatal day $1(\mathrm{P} 1)$ to $\mathrm{P} 21$. On $\mathrm{P} 7$, the pups were exposed to $6 \mathrm{~h}$ of isoflurane $(0.75 \%)$ anaesthesia. Neuroapoptosis was examined using the TUNEL assay. The expression of cleaved caspase-3, the apoptotic pathway proteins (Bad, Bax, Bcl-2 and Bcl-xL), the phosphoinositide 3-kinase (PI3K)/protein kinase $\mathrm{B}(\mathrm{Akt})$ pathway proteins [Akt, phosphorylated (-)Akt, glycogen synthase kinase $3 \beta$ (GSK-3 $\beta$ ), p-GSK-3 $\beta$, phosphatase and tensin homolog (PTEN)] and nuclear factor- $\kappa \mathrm{B}(\mathrm{NF}-\kappa \mathrm{B})$-mediated signalling proteins were determined by western blot analysis. General behaviour, as well as the learning ability and memory of the pups were assessed. Naringenin significantly inhibited isoflurane-induced neuroapoptosis and markedly decreased the protein expression of caspase-3, Bad, Bax, NF- $\kappa \mathrm{B}$, tumor necrosis factor- $\alpha$, interleukin (IL)-6 and IL-1 $\beta$. Furthermore,
\end{abstract}

Correspondence to: DrGuo-Hai Xu, Department of Anesthesiology, TheSecondAffiliatedHospitalof NanchangUniversity,1 MingdeRoad, Nanchang, Jiangxi 330006, P.R. China

E-mail: guohaiinj@hotmail.com

${ }^{*}$ Contributed equally

Key words: anaesthesia, isoflurane, naringenin, nuclear factor- $\kappa \mathrm{B}$, neuroapoptosis, phosphoinositide 3/protein kinase B/phosphatase and tensin homolog signalling pathway naringenin increased the expression of Bcl-xL and Bcl-2 and activated the PI3K/Akt pathway. Significant improvements in learning capacity and memory retention were observed following naringenin treatment. Naringenin effectively ameliorated cognitive dysfunction and reduced isoflurane-induced apoptosis as well as modulating the PI3/Akt/PTEN and NF- $\kappa \mathrm{B}$ signalling pathways.

\section{Introduction}

General anaesthetics are commonly employed in operative settings for surgery and/or for pain relief. Volatile anaesthetics such as isoflurane and sevoflurane are widely used in adults as well in infants and young children for surgical procedures and other medical treatments that require anaesthesia (1). Previous findings have demonstrated that prolonged exposure to the anaesthetics isoflurane and sevoflurane leads to apoptotic neurodegeneration in developing animal brains and also affects learning and memory (2-7). Liang et al (8) reported that isoflurane anaesthesia induced more robust apoptosis of cells in the neonatal rat brain than sevoflurane when administered at an eqivalent dose. Further clinical retrospective studies have demonstrated that children $<4$ years of age exposed to anaesthesia are possibly at a higher risk of developing learning disabilities $(9,10)$.

The increased expression of activated caspase- 3 , the principal enzyme in the cellular apoptotic pathway, and $\beta$-amyloid peptide $(\mathrm{A} \beta)$ levels were observed in murine brains following isoflurane exposure $(11,12)$. Studies have also suggested that inflammation may possibly be involved in anaesthetic-mediated neurodegeneration, particularly cognitive dysfunction $(13,14)$. Wu et al (15) observed that isoflurane increased pro-inflammatory cytokine levels in both in vitro and in vivo experiments. Inflammation involving microglial activation and increased levels of pro-inflammatory cytokines, particularly tumor necrosis factor- $\alpha$ (TNF- $\alpha$ ) and interleukin (IL)- 6 in the brain, may lead to cognitive impairment (16-21).

It is well documented that the nuclear factor- $\kappa \mathrm{B}$ $(\mathrm{NF}-\kappa \mathrm{B})$-dependent signalling pathway is required for cytokine gene transcription (22). The NF- $\kappa \mathrm{B}$ family of 
transcription factors play essential roles in inflammation and innate immunity, and regulate many genes, particularly those involved in inflammation, injury and stress (23). Isoflurane has been reported to induce neuronal apoptotic degeneration via $\left[\mathrm{Ca}^{2+}\right]_{\mathrm{i}}$ overload through gamma-aminobutyric acid $(\mathrm{GABA})_{\mathrm{A}}$ receptor-mediated synaptic voltage-dependent calcium channels (VDCCs). The excessive release of $\mathrm{Ca}^{2+}$ from the endoplasmic reticulum through the activation of inositol-1,4,5-trisphosphate (IP3) receptors $(24,25)$, activates the $\mathrm{NF}-\kappa \mathrm{B}$ signalling pathway (26-28), eventually leading to increased levels of pro-inflammatory cytokines $(27,29)$.

In addition, the phosphoinositide 3-kinase (PI3K)/protein kinase $\mathrm{B}(\mathrm{Akt})$ pathway is widely expressed in the central nervous system and affected by growth factors, such as nerve growth factor, brain-derived neurotrophic factor and glial cell line-derived neurotrophic factor, as well as cytokines and neurotransmitters. The pathway plays a crucial role in neuronal survival (30). Akt, a serine/threonine kinase, regulates neuronal survival and is activated by growth factors (31). On activation, Akt inhibits apoptosis by phosphorylating and inactivating Bad and glycogen synthase kinase $3 \beta$ (GSK-3 $\beta$ ) (32-34). Another target modulated by Akt is $\mathrm{NF}-\kappa \mathrm{B}$ (35). Thus, targeting the PI3/Akt pathway may significantly modulate neuronal cell survival and inflammation.

The flavonoid naringenin is found in oranges, grapefruits and tomatoes (36). It has been proven to possess health benefits such as anti-inflammatory, antioxidant and free radical scavenging properties $(37,38)$ as well as to reduce lipid levels (39) and to prevent dyslipidemia (40). Taking into consideration the biological effects of naringenin, in the present study, we examined the ability of naringenin to inhibit isoflurane-induced neuroapoptosis and to ameliorate inflammation-induced cognitive dysfunction.

\section{Materials and methods}

Experimental animals. The study was approved by the Institutional Animal Care Committee of Nanchang University (Nanchang, China) and was performed in accordance with the National Institutes of Health Guidelines for the Use of Laboratory Animals. Pregnant Sprague-Dawley rats (Guangdong Medical Laboratory Animal Center, Guangzhou, China) were housed separately in sterile cages under animal room conditions maintained at $22 \pm 1^{\circ} \mathrm{C}$ on a 12 -h light/dark cycle. The rats were provided with standard pelleted diet and water ad libitum and closely monitored for the date of birth of the pups, that was noted as postnatal day 0 (P0). The pups were maintained carefully with free access to water with their littermates.

Chemicals and reagents. Isoflurane $(0.75 \%)$ and naringenin were obtained from Sigma-Aldrich (St. Louis, MO, USA). The following antibodies were used for expression analysis: antibodies against cleaved caspase-3 (\#9661), Bcl-2 (mouse $\mathrm{mAb}$ \#15071), Bad (rabbit mAb \#9268), Bcl-xL (rabbit mAb \#2764), Bax (rabbit mAb \#5023), Akt (rabbit mAb \#4691), phosphorylated (p-)Akt (rabbit mAb \#4060), GSK-3 $\beta$ (rabbit mAb \#9315), p-GSK-3 $\beta$ (rabbit mAb \#9322), phosphatase and tensin homolog (PTEN; rabbit mAb \#9188), the inhibitors of apoptosis proteins (IAP) xIAP (rabbit mAb \#2045), cIAP-1 (rabbit mAb \#3130), survivin (rabbit mAb \#2808),
TNF- $\alpha$ [rabbit mAb (mouse specific) \#11948] and $\beta$-actin (mouse mAb \#3700) (all from Cell Signalling Technology,

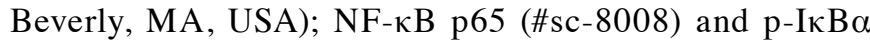
(\#sc-8404) (both from Santa Cruz Biotechnology, Santa Cruz, CA, USA); and IL-6 (ab100712) and IL-1ß (ab197742) (both from Abcam, Cambridge, MA, USA). All other chemicals used were of analytical grade and purchased from Sigma-Aldrich, unless specified otherwise.

Anaesthetic exposure and dosing. Sixty pups were used and 12 pups were randomly allocated to each group. Separate groups of pups were administered naringenin $[25,50$ or $100 \mathrm{mg} / \mathrm{kg}$ body weight (b.wt], orally from P3 until P21 along with a standard diet. On P7, pups weighing about 16-20 g were exposed to $0.75 \%$ isoflurane [approximately 0.3 minimum alveolar concentration (MAC)] (41) in $30 \%$ oxygen or air in a temperature controlled chamber for $6 \mathrm{~h}(42,43)$. P7 rats were selected on the basis of previous studies suggesting that this period was most vulnerable to anaesthesia-induced neuronal damage (2). The control rats received no anaesthesia or naringenin. At the end of $6 \mathrm{~h}$ of isoflurane exposure, the rat pups were sacrificed and brain tissues were excised in order to perform protein expression analysis and the TUNEL assay. Briefly, P7 rat pups were anaesthetized with isoflurane and transcardially perfused with ice-cold saline followed by $4 \%$ paraformaldehyde in $0.1 \mathrm{M}$ phosphate buffer. The brain tissues were excised and embedded in paraffin. The rat pups not exposed to either anaesthesia or treated with naringenin were segregated as control rats. The isoflurane-exposed pups not administered naringenin were grouped as anaesthetic control pups. The animals were anesthetised with pentobarbital (40 mg/kg body weight) and were intracardially perfused with $0.01 \mathrm{M}$ PBS. The brains were immediately excised and the hippocampi were isolated. The brain tissues were kept at $-80^{\circ} \mathrm{C}$ until required for use in subsequent experiments.

Assessment of neuroapoptosis by TUNEL assay. Following $6 \mathrm{~h}$ of exposure to isoflurane, neuroapoptosis was assessed by the TUNEL assay as previously described by Li et al (44). Three sections $5 \mu \mathrm{m}$ thick were sliced $(200 \mu \mathrm{m}$ apart) on the same plane of the hippocampus from each rat pup and were further analysed. Apoptosis was assessed using a DeadEnd ${ }^{\mathrm{TM}}$ fluorometric TUNEL System kit (Promega, Madison, WI, USA). The slides were carefully protected from exposure to direct light during the experiment. The Hoechst stain was used to stain nuclei. The TUNEL-positive cells in the regions of hippocampal CA1, CA3 and dentate gyrus (DG) were further analysed using NIS-Elements Basic Research (BR) imaging processing and analysis software (Nikon Corporation, Tokyo, Japan).

Immunohistochemistry (IHC) staining. Cleaved caspase-3 expression was used as a marker of apoptosis and cell death. Immunohistochemical analysis was performed to assess caspase-3 expression in the hippocampi of isoflurane-exposed rats as previously described by Li et al $(42,44)$. Briefly, the brain sections were incubated with anti-cleaved caspase- 3 primary antibody overnight at $4^{\circ} \mathrm{C}$ and further incubated with a secondary antibody (Santa Cruz Biotechnology) for $40 \mathrm{~min}$. Following treatment with avidin-biotinylated peroxidase complex (Vectastain ABC-kit; Vector Laboratories, 
Burlingame, CA, USA) for $40 \mathrm{~min}$, the tissue sections were treated with diaminobenzidine and were analysed with NIS-Elements BR imaging processing and analysis software. The density of cleaved caspase-3-positive cells in CA1, CA3 and $\mathrm{DG}$ regions was calculated by dividing the number of caspase-3-positive cells by the area of that brain region.

Western blot analysis. Protein expression in the hippocampi of rat pups was examined in order to determine the effect of naringenin on the outcome of isoflurane exposure according to previously described procedures $(42,43)$. Briefly, the tissues were homogenised and proteins were isolated. Protein concentrations in the tissue samples were determined using a BCA protein assay (Bio-Rad, Hercules, CA, USA). Sixty micrograms of each protein sample were subjected to SDS-PAGE separation and blotted on to polyvinylidene difluoride membranes. The blots were incubated with primary antibodies overnight at $4^{\circ} \mathrm{C}$ and washed and further incubated with appropriate secondary antibodies. The immunoreactive bands were visualized and the images were scanned using an ImageMaster II scanner (GE Healthcare, Milwaukee, WI, USA). The densities of the bands were further analysed by ImageQuant TL software v2003.03 (GE Healthcare). Protein expression was normalized to $\beta$-actin.

Memory and learning studies. Isoflurane induction has been documented to cause cognitive dysfunction affecting learning and memory (2). In the present study, the effects of naringenin on the behaviour and learning of rat pups were assessed.

Open-field test. An open-field test was performed to assess the emotional response of the rats to a novel environment. P42 rats exposed to isoflurane on $\mathrm{P} 7$ were subjected to an open-field test as previously described (45). The response was noted as the movement of the rats in the open field. The total distance travelled in meters over $10 \mathrm{~min}$ was recorded. The movement of the animals was monitored and analysed using a computerized video tracking system (SMART; Panlab S.L., Barcelona, Spain).

Elevated plus-maze test. The elevated plus-maze test was performed to evaluate anxiety-related behaviour in rodents. The test was conducted according to the procedure described by Satoh et al (45). The apparatus consisted of two open $(25 \times 5 \mathrm{~cm})$ and two closed arms. The arms were placed at $50 \mathrm{~cm}$ above the floor. The responses of the animals were recorded using a computerized video tracking system (SMART). The behaviour of $\mathrm{P} 42$ rats was observed for a period of $10 \mathrm{~min}$. The percentage of time spent in the open arm was noted and this was considered as an anxiety index.

$Y$-maze test. This study was performed to assess spatial working memory and was performed as previously described by Satoh et al (45). The symmetrical, acrylic Y-shaped maze consisted of three arms $(25 \times 5 \mathrm{~cm})$ that were separated by $15-\mathrm{cm}$-high transparent walls. The rats were placed at the centre of the maze and each rat was allocated $8 \mathrm{~min}$ to freely explore the maze. The total number of arms entered and sequence of entry were noted too. The calculations were performed as described by Kodama et al (46).

Fear conditioning test. The $\mathrm{P} 49$ rats were subjected to a fear response test. The fear conditioning test is a reliable, sensitive test used to assess the effect of a hippocampal-dependent and hippocampal-independent conditioned stimulus-unconditioned stimulus pair that were separated by 1 min each. The unconditioned stimulus consisted of $1 \mathrm{~mA}$ foot shock of a $1 \mathrm{sec}$ duration whereas the conditioned stimulus consisted of $80 \mathrm{~dB}$ of white noise for $20 \mathrm{sec}$. The unconditioned stimulus was delivered during the last few seconds of the conditioned stimulus. A contextual fear test was performed in the conditioning chamber for a period of $5 \mathrm{~min}$ in the absence of white noise $24 \mathrm{~h}$ after conditioning. A cued test (for the same set of rats) was conducted by presenting a cue ( $80 \mathrm{~dB}$ white noise, 3-min duration) while presenting distinct visual and tactile cues. The movement of the animals was monitored using the computerized video tracking system (SMART). The freezing response rate of the rats, which was identified as the absence of movement in any part of the body for $1 \mathrm{sec}$, was scored automatically and the data were analysed and used as a measure of fear memory (4).

Morris water maze (MWM) test. The effect of naringenin on isoflurane-induced alterations in memory and learning, spatial reference memory and learning were evaluated using the MWM and experiments were performed as described previously by Li et al (44).

The P7 rat pups that were exposed to isoflurane and/or naringenin were trained for a period of 4 days between P31 and P35 in the MWM. A platform $10 \mathrm{~cm}$ in diameter was submerged in a circular pool $(200 \mathrm{~cm}$ diameter, $60 \mathrm{~cm}$ depth) that was filled with warm water $\left(23 \pm 2^{\circ} \mathrm{C}\right)$. The rats were subjected to 2 training sessions a day. The rats were allowed $60 \mathrm{sec}$ to locate the hidden platform in the pool. If the animals were unable to locate the platform within $60 \mathrm{sec}$, they were gently guided. The performance of the rats and swim paths were recorded using the ANY-maze video tracking system (Stoelting Co., Wood Dale, IL, USA). The tracking system records and measures the time taken (latency) by each rat to find the platform(s), and also information on other behaviours.

P35 rats were subjected to cued trials to test for any non-cognitive performance impairments such as visual impairments and/or swimming difficulties. The circular pool was covered by a white cloth in order to conceal the visual cues. The rats were subjected to 4 trials per day. During each trial, the animals were placed in a specific position in the swimming pool and allowed to swim to the platform which had a rod attached, that served as the cue. The rod was placed approximately at $20 \mathrm{~cm}$ above water level in any one of the four quadrants of the swimming pool. The rats were given a time of $60 \mathrm{sec}$ to locate the submerged platform with the help of the cue and a time of $30 \mathrm{sec}$ to sit on the platform. The rats that was unable to locate the platform in the given period of time, were gently guided and allowed to remain there for $30 \mathrm{sec}$. The time taken by each animal to find the cued platform was recorded.

Place trials were also performed. The curtains that surrounded the pool for the cued trials were removed and the same set of rats were assessed for their ability to learn the spatial relationship between cues and the submerged platform (with no cue rod), that was kept in one of the four quadrants. The platform was placed in the same position throughout the place trials. The rats were placed at random starting points and the time taken to reach the platform was noted.

Furthermore, the rats were subjected to probe trials in order to assess memory. The probe trials were conducted $24 \mathrm{~h}$ after place trials. The submerged platform with no cue rod was removed from the target quadrant (the quadrant in which the 
platform was placed throughout the place trials). Rats were allowed to swim for $60 \mathrm{sec}$ and the time that each rat spent in the target quadrant looking for the submerged platform was recorded. The data are presented as the percentage of time spent in the quadrants. The time spent in the target quadrant by the rats in comparison with the time spent in other quadrants is used as an indication of memory retention.

Statistical analysis. The results are presented as the means $\pm \mathrm{SD}$, taken from three or six independent experiments. The data were subjected to statistical analysis using the SPSS statistical package (22.0). The values at $\mathrm{p}<0.05$ were considered to indicate a statistically significant difference as determined by one-way analysis of variance (ANOVA) at $\mathrm{p}<0.05$ followed by Duncan's multiple range test (DMRT) for post hoc analysis.

\section{Results}

Naringenin reduces isoflurane-induced neuroapoptosis. Previous studies have demonstrated that isoflurane exposure induces neuroapoptosis $(5,47)$. In agreement with the findings of earlier studies, in our study, an increase $(\mathrm{p}<0.05)$ in apoptotic cell counts was observed following $6 \mathrm{~h}$ of isoflurane exposure in the CA1, CA3 and the DG regions or the rat hippocampi (Fig. 1). However, the administration of naringenin caused a marked decline in TUNEL-positive cell counts indicating that it effectively provided protection to the neuronal cells exposed to anaesthesia. Naringenin at $100 \mathrm{mg}$ exhibited maximum protective effects as compared with lower doses of 25 and $50 \mathrm{mg}$.

Cleaved caspase- 3 expression is considered as a significant marker of apoptosis $(2,6)$. To determine whether naringenin modulated the expression of caspase- 3 in the hippocampus, we assessed the expression of cleaved caspase- 3 by IHC staining. An increase in the number of caspase-positive cells was observed in the hippocampus of rats exposed to isoflurane (Fig. 2). Analysis of cleaved caspase-3 protein expression by western blot analysis also revealed significantly enhanced expression $(\mathrm{p}<0.05)$ following $6 \mathrm{~h}$ of isoflurane exposure (Fig. 3). Elevated caspase- 3 expression may have contributed to the enhanced apoptotic counts evaluated by the TUNEL assay. Notably, naringenin at doses of 25, 50 and $100 \mathrm{mg}$ downregulated caspase-3 protein expression and caspase-3-positive cell counts which was in agreement with the TUNEL-positive cell counts. Furthermore, caspase-3 expression was found to be dosedependent with a $100 \mathrm{mg}$ dose of naringenin producing the greatest decline in caspase-3 expression.

Naringenin effectively modulates the expression of apoptotic pathway proteins. Isoflurane has been reported to cause robust neurodegeneration and apoptosis in the developing brain (5). The balance between Bcl-2 pro-apoptotic proteins (Bax and Bad) and anti-apoptotic proteins (Bcl-2 and $\mathrm{Bcl}-\mathrm{xL}$ ) regulates mitochondrial membrane integrity and the release of apoptogenic factors that critically affect cell survival and death (48). Isoflurane exposure for $6 \mathrm{~h}$ in $\mathrm{P} 7$ rat pups caused a significant increase in the expression of Bax and Bad with a decrease in the expression of Bcl-2 and Bcl-xL (Fig. 3). The administration of naringenin at doses of 50 and $100 \mathrm{mg}$ induced a marked downregulation of the expression of Bax and Bad and significantly $(\mathrm{p}<0.05)$ elevated the expression of the anti-apoptotic proteins $\mathrm{Bcl}-\mathrm{xL}$

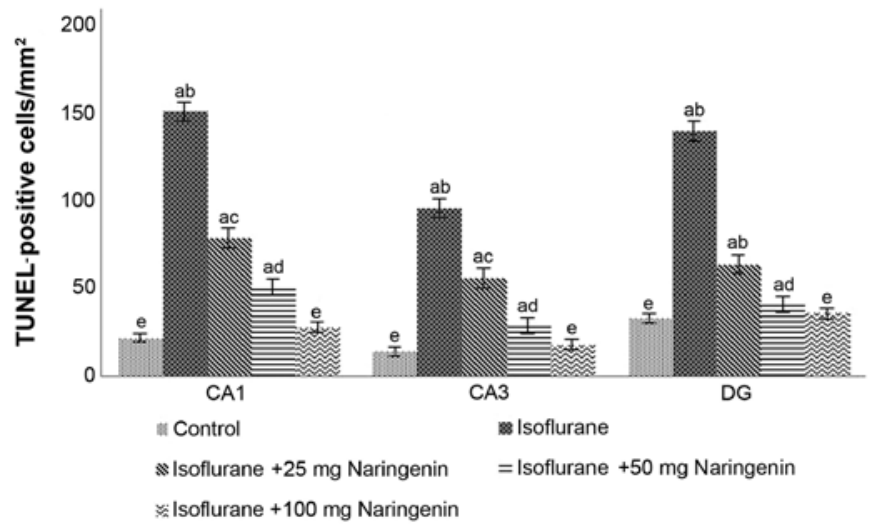

Figure 1.Effect of naringenin on isoflurane-induced neuroapoptosis. Naringenin effectively reduced neuroapoptosis in the hippocampus of isoflurane-exposed rat pups. Values are presented as the means $\pm S D, n=6$. a, $p<0.05$ compared with the control; b-f, represents mean values within the same group that differ from each other at $\mathrm{p}<0.05$ as determined by one-way ANOVA followed by Duncan's multiple range test analysis.

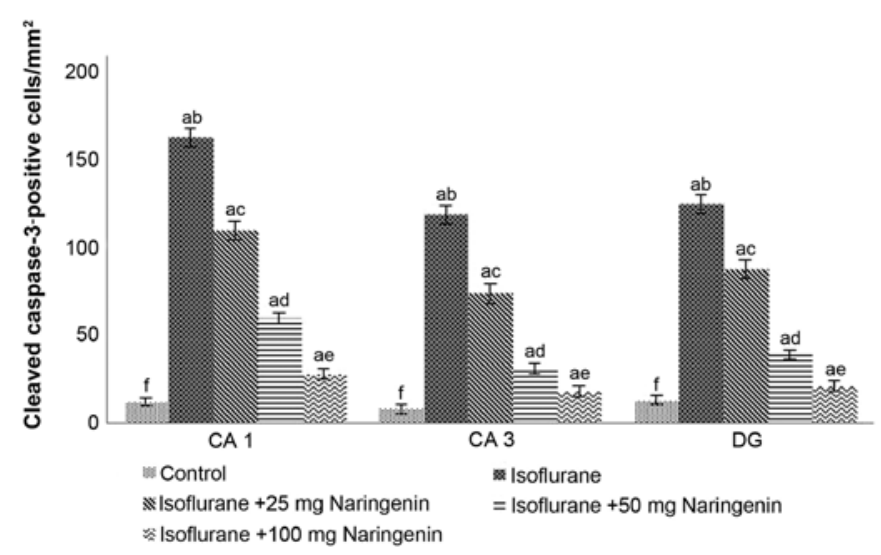

Figure 2. Naringenin reduces the expression of cleaved caspase-3. Cleaved caspase- 3 expression was markedly reduced by naringenin in a dose-dependent manner, thus reducing apoptosis. Values are presented as the means $\pm \mathrm{SD}, \mathrm{n}=6$. $\mathrm{a}, \mathrm{p}<0.05$ compared with the control; $\mathrm{b}-\mathrm{f}$, represents mean values within the same group that differ from each other at $\mathrm{p}<0.05$ as determined by one-way ANOVA followed by Duncan's multiple range test analysis.

and Bcl-2. Although a $25 \mathrm{mg}$ dose of naringenin modulated protein expression, the higher doses produced more pronounced effects. Taken together, these findings suggest that naringenin effectively regulates the expression of the apoptotic pathway proteins in a dose-dependent manner.

Naringenin potentially activates the PI3K/Akt pathway. Pro-survival pathways such as the PI3K/Akt pathway may be inactivated during the apoptotic process (33). The pathway is widely expressed in developing brains. In our study, isoflurane anaesthesia suppressed the pathway as the levels of Akt, p-Akt, GSK-3 $\beta$ and p-GSK-3 $\beta$ were reduced in the rat pups exposed to anaesthesia and not treated with naringenin. Treatment with naringenin significantly upregulated $(\mathrm{p}<0.05)$ the protein levels of Akt, p-Akt, and p-GSK-3 $\beta$. However, GSK-3 $\beta$ expression was not markedly altered by the administration of naringenin (Fig. 4). PTEN, the negative regulator of the pathway, was increased by isoflurane. Naringenin caused a significant decline in the expression of PTEN in a 

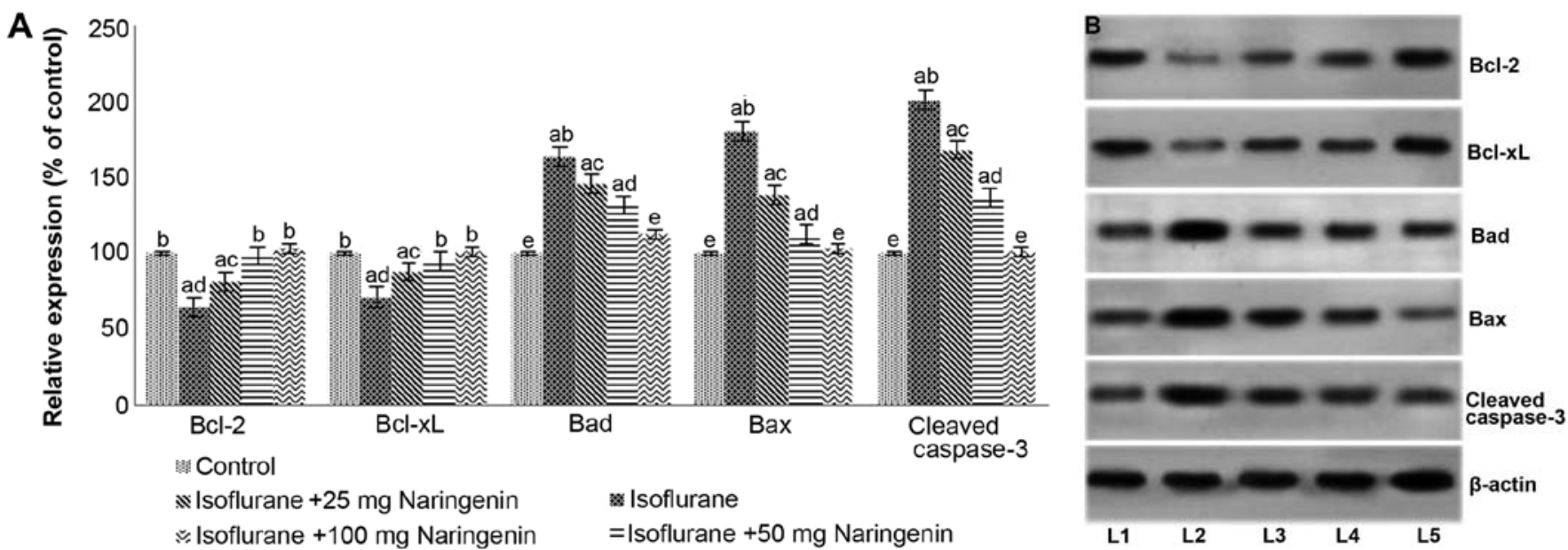

Figure 3. Naringenin modulates the expression of apoptosis pathway proteins. (A) Quantitative analysis of relative protein expression. Naringenin effectively regulated the expression of anti-apoptotic and pro-apoptotic proteins; naringenin enhanced the expression of Bcl-2 and Bcl-xL,and markedly downregulated the expression of Bax, Bad and caspase-3. (B) Representative western blots of the proteins. Values are presented as the means $\pm \mathrm{SD}, \mathrm{n}=6$. a, $\mathrm{p}<0.05$ compared with the control; b-f, represent mean values within the same group that differ from each other at $\mathrm{p}<0.05$ as determined by one-way ANOVA followed by Duncan's multiple range test analysis. (L1, control; L2, isoflurane; L3, isoflurane $+25 \mathrm{mg}$ naringenin; $\mathrm{L} 4$, isoflurane $+50 \mathrm{mg}$ naringenin; $\mathrm{L} 5$, isoflurane $+100 \mathrm{mg}$ naringenin).
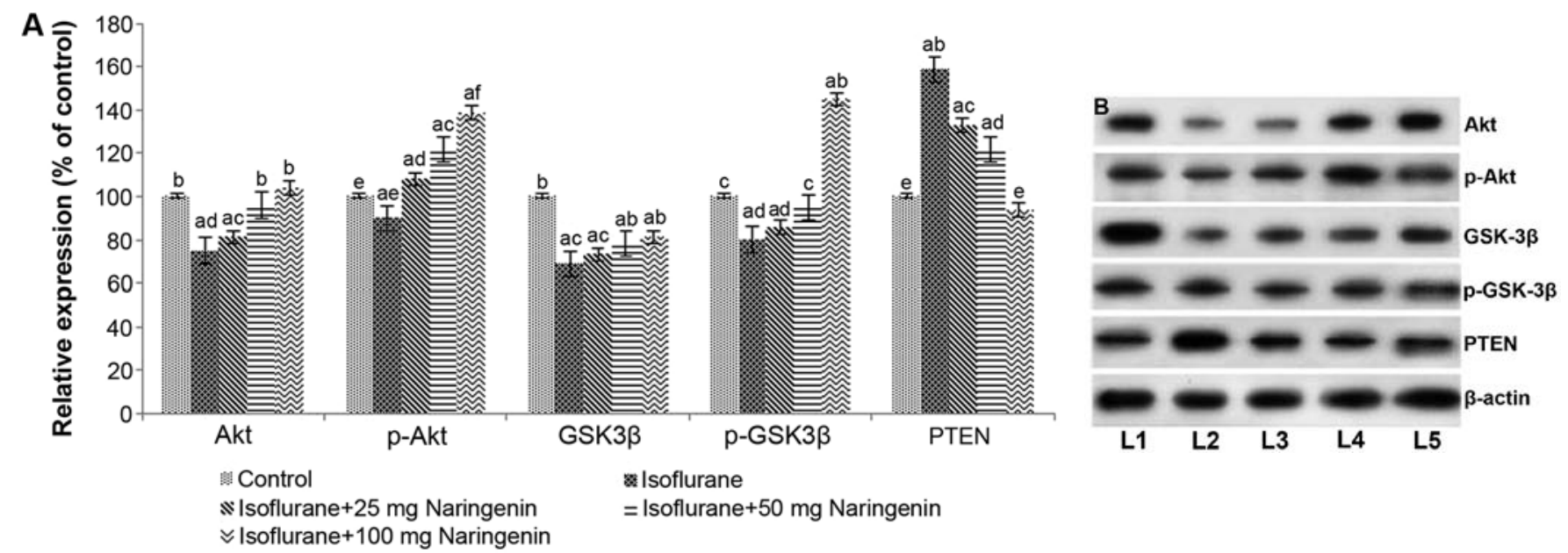

Figure 4. Naringenin modulates the phosphoinositide 3-kinase (PI3K)/protein kinase B (Akt) signalling pathway. (A) Quantitative analysis of relative protein expression. Naringenin (25,50 and $100 \mathrm{mg}$ ) potentially activated the PI3K/Akt pathway. (B) Representative western blots of the proteins. Values are presented as the means $\pm \mathrm{SD}, \mathrm{n}=6$. a, $\mathrm{p}<0.05$ compared with the control; $\mathrm{b}$-f, represents mean values within the same group that differ from each other at $\mathrm{p}<0.05$ as determined by one-way ANOVA followed by Duncan's multiple range test analysis. (L1, control; L2, isoflurane; L3, isoflurane $+25 \mathrm{mg}$ naringenin; $\mathrm{L} 4$, isoflurane $+50 \mathrm{mg}$ naringenin; L5, isoflurane $+100 \mathrm{mg}$ naringenin).

dose-dependent manner with maximal effects observed at a dose of $100 \mathrm{mg}$ naringenin.

Naringenin significantly regulates the $N F-\kappa B$ signalling pathway. The commonly used anaesthetic isoflurane is known to induce neuro-inflammation (15). It has been reported that the isoflurane-induced elevated cytosolic $\mathrm{Ca}^{2+}$ levels activate the $\mathrm{NF}-\kappa \mathrm{B}$ signalling pathway $(28,29)$. Activated $\mathrm{NF}-\kappa \mathrm{B}$ is capable of further activating and regulating various genes involved in inflammatory responses (11). We observed significantly high

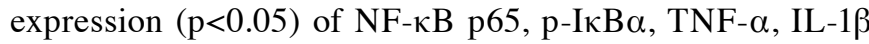
and IL- 6 following isoflurane exposure. However, the levels of XIAP and cIAP were reduced (Fig. 5) which contributes to increased apoptosis. Naringenin at all the tested doses caused marked increases in the expression of XIAP and cIAP.
However, naringenin suppressed the activation of NF- $\kappa \mathrm{B}$ and subsequently inhibited the expression of TNF- $\alpha$, IL- $1 \beta$ and IL-6.

Effects of naringenin on the behaviour and memory of rats exposed to inhalation anaesthesia. The behaviour of the animals exposed to isoflurane was assessed. In the present study, the rat pups exposed to isoflurane on $\mathrm{P} 7$ were subjected to an open-field test which involved observing the behaviour of the pups in a novel environment. Isoflurane only-exposed pups did not exhibit noticeable behavioural disturbances; the distance they moved across the new field was comparatively less $(p<0.05)$ than that in the rats exposed to anaesthesia and treated with naringenin. The rats that received 50 and $100 \mathrm{mg}$ doses of naringenin exhibited behaviour similar 

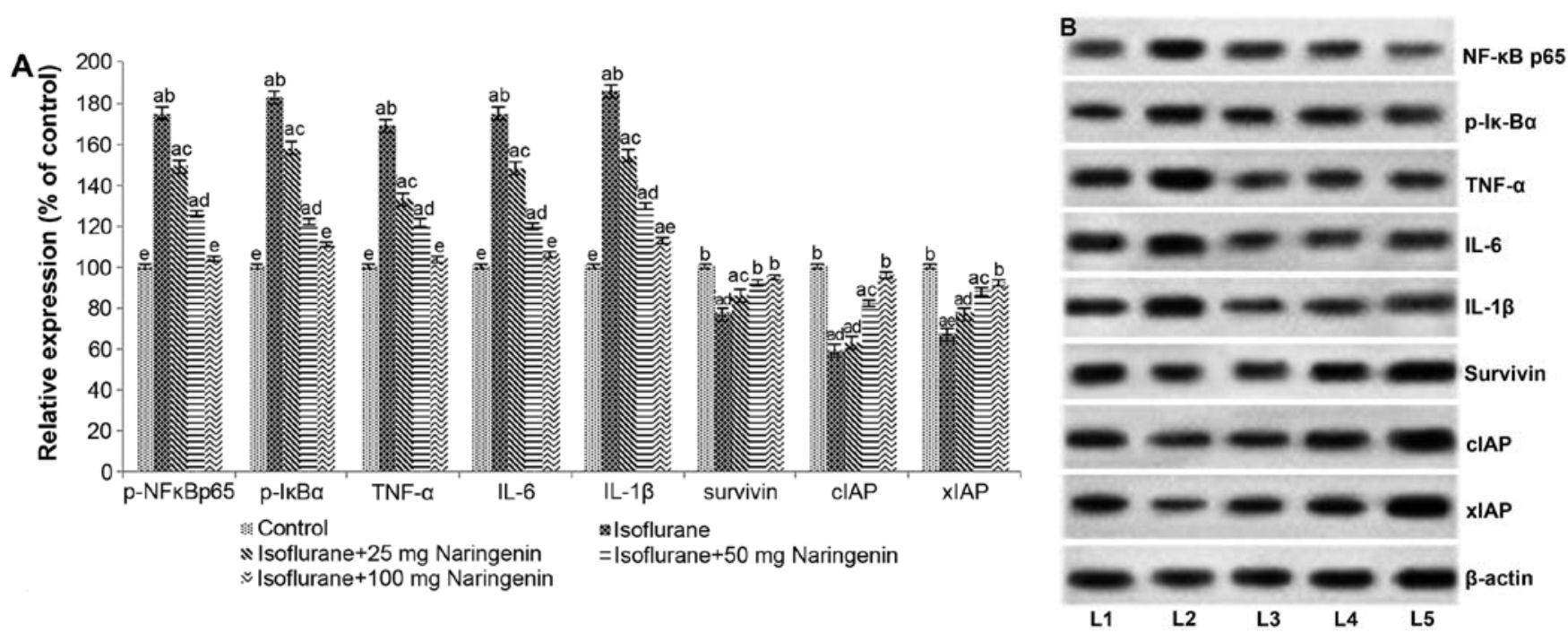

Figure 5. Naringenin modulates the nuclear factor- $\mathrm{kB}(\mathrm{NF}-\mathrm{\kappa} B$ ) signalling pathway. (A) Quantitative analysis of relative protein expression. Naringenin caused marked inhibition of NF- $\mathrm{kB}$ signalling leading to reduced transcription of cytokines and inflammatory mediators and the increased expression of inhibitors of apoptosis proteins. (B) Representative western blots of the proteins. Values are presented as the means $\pm \mathrm{SD}, \mathrm{n}=6$. a, $\mathrm{p}<0.05$ compared with the control; b-f, represents mean values within the same group that differ from each other at $\mathrm{p}<0.05$ as determined by one-way ANOVA followed by Duncan's multiple range test analysis. (L1, control; L2, isoflurane; L3, isoflurane $+25 \mathrm{mg}$ naringenin; $\mathrm{L} 4$, isoflurane $+50 \mathrm{mg}$ naringenin; L5, isoflurane $+100 \mathrm{mg}$ naringenin).
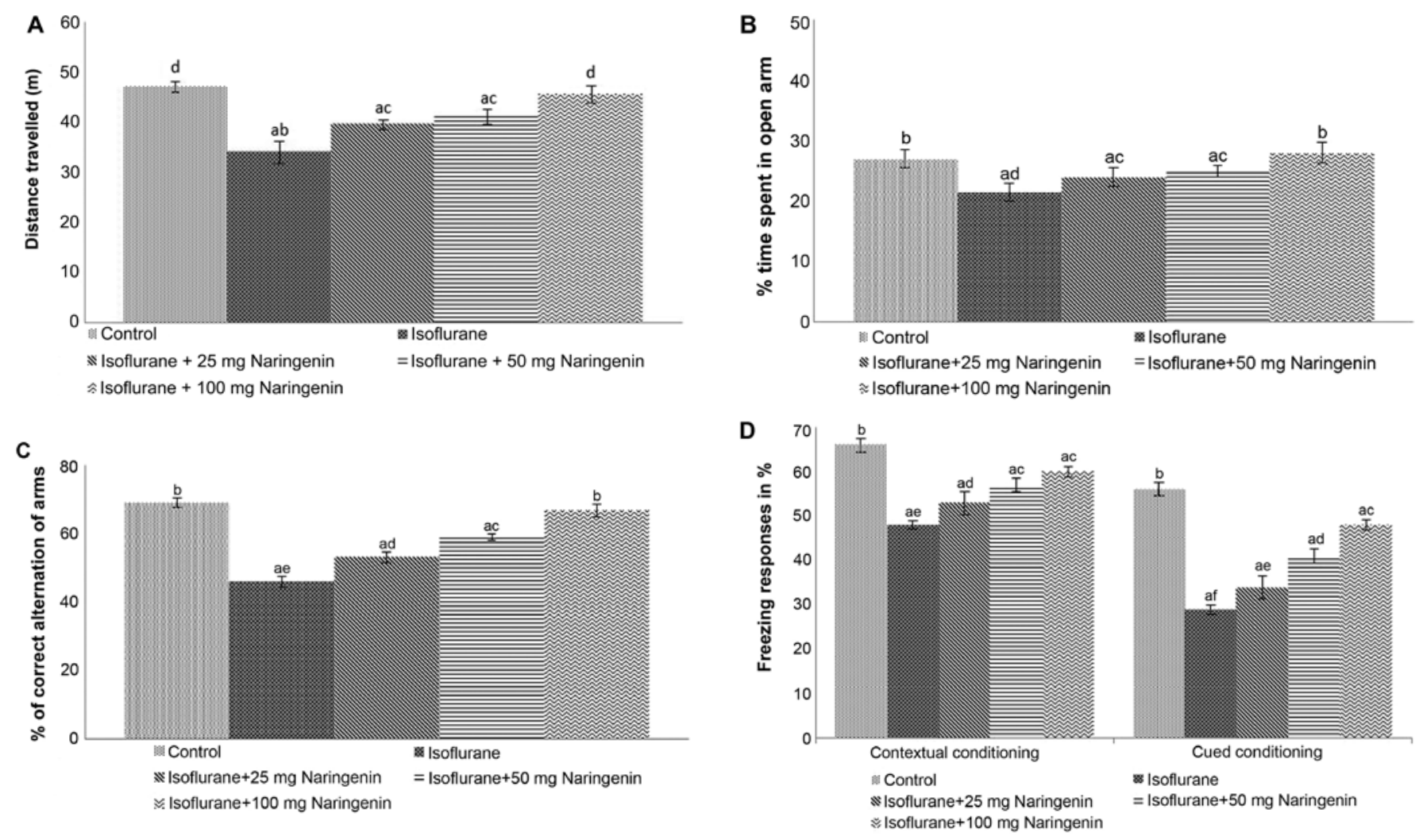

Figure 6. Naringenin improves the cognition and memory of rats following exposure to isoflurane on postnatal day 7 (P7). Behaviour of P42 rats (A) in a novel environment, (B) an elevated maze, (C) in a Y-maze and (D) the freezing response of the rats subjected to contextual and cued fear conditioning. Values are presented as the means $\pm S D, n=6$. a, $p<0.05$ compared with the control; $b$-f, represents mean values within the same group that differ from each other at $p<0.05$ as determined by one-way ANOVA followed by Duncan's multiple range test analysis.

to the control rats that received no anaesthesia or naringenin (Fig. 6A). In order to assess anxiety-related behaviour, an elevated plus-maze test was performed. The percentage of time spent in the open arms was noted. Considerable differences were observed between the behaviour of the animals treated with naringenin compared with that of the animals in the isoflurane control groups (Fig. 6B).

Working memory is involved in holding information temporarily to perform cognitive tasks that are complex and it involves both the hippocampus and prefrontal cortex $(49,50)$. 

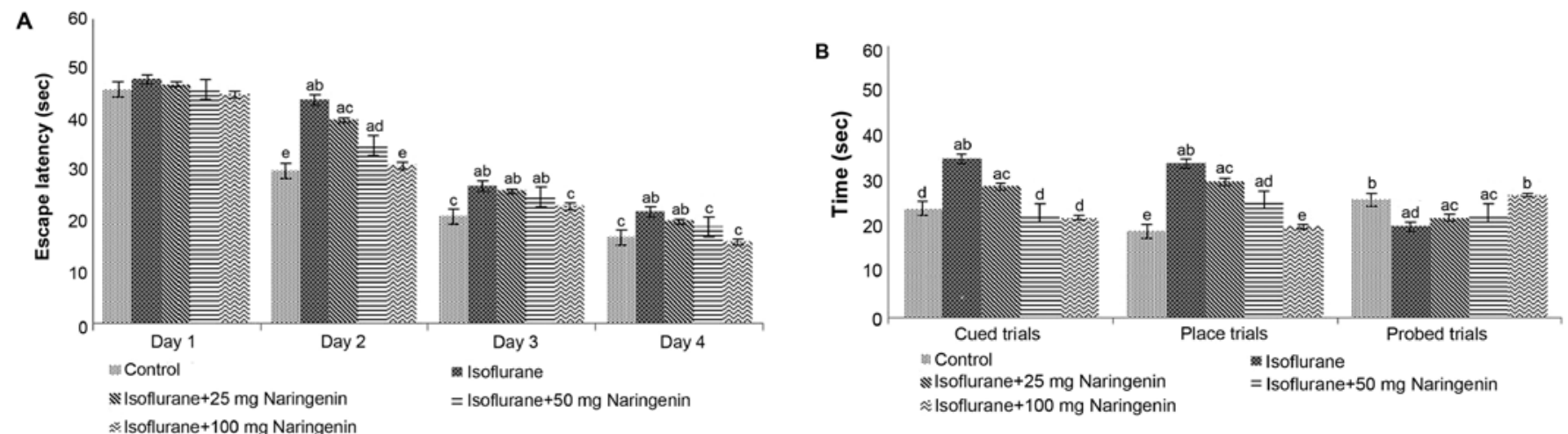

Figure 7. Assessment of learning and memory of postnatal day 35 (P35) rats following exposure to anesthesia on P7 by the Morris water maze (MWM) test. Escape latency of the rats during (A) the training period and (B) cued, place and probe trials with the MWM. Values are presented as the means \pm SD, $n=6$. a, $\mathrm{p}<0.05$ compared with the control; $\mathrm{b}-\mathrm{f}$, represents mean values within the same group that differ from each other at $\mathrm{p}<0.05$ as determined by one-way ANOVA followed by Duncan's multiple range test analysis.

To determine whether exposure to isoflurane affected spatial working memory, a Y-maze test was conducted. The tasks examined whether the rats remembered the arm selected in the preceding choice. Rat pups exposed to isoflurane exhibited significantly indifferent behaviour as compared with the control rats not exposed to isoflurane. Naringenin at all the three tested doses considerably improved the performance of the animals (Fig. 6C).

The freezing responses of the animals exposed to isoflurane were significantly $(\mathrm{p}<0.05)$ reduced in both the contextual and cued conditioning tests (Fig. 6D). The freezing responses exhibited by the animals treated with naringenin were substantially higher than those of the animals administered with isoflurane alone.

Assessment of learning and memory of animals using MWM tests. To further evaluate the effect of isoflurane exposure on potential learning and memory deficits, the animals were subjected to the MWM test. The MWM is the most frequently used and reliable measure of hippocampus-dependent spatial navigation and reference memory assessment (51). The rats exposed to isoflurane anaesthesia on P7 were trained to explore the swimming pool and reach the hidden submerged platform. The time taken by each animal to find and reach the platform was recorded as the escape latency. The latency time recorded was found to decrease with each training session in all the animals irrespective of whether they were supplemented with naringenin or not (Fig. 7A). Nevertheless, the isoflurane only-treated group exhibited slight variations from the animals that received naringenin and isoflurane exposure on P7.

The cued trials were conducted by hiding the visual cues in order to evaluate swimming and visual abilities. The P35 rats exposed to isoflurane anaesthesia on $\mathrm{P} 7$ took considerably longer to reach the submerged platform with the rod compared with the control group of rats that had received no anaesthesia. Naringenin treatment at doses of 50 and $100 \mathrm{mg}$ was observed to improve the performance of the rats; however, the results were not significant at a dose of $25 \mathrm{mg}$ naringenin. The animals that received naringenin $(50$ and $100 \mathrm{mg}$ ) took less time to reach the platform compared with the rats that had received isoflurane alone. Furthermore, $100 \mathrm{mg}$ naringenin produced the maximum improvement in the performance of the rats (Fig. 7B). Place and probe trials were conducted to assess the ability of the rats to discover and remember the location of the submerged platform without the cue rod. In the place trials, the naringenin-treated rats exhibited a significant improvement $(\mathrm{p}<0.05)$ in performance. The rats took significantly less time to reach the platform.

Memory retention was assessed by removing the platform from the target quadrant and placing it in a random quadrant other than the target quadrant. The ability of the rats to look for the platform in the target quadrant was observed. Isoflurane exposure was found to have a significant impact on the memory of the rats, as animals exposed to isoflurane spent significantly less time $(\mathrm{p}<0.05)$ in the target quadrant in comparison with the control group not exposed to anaesthesia (Fig. 7B). The observed results suggest that isoflurane induced memory and learning impairments. Naringenin administration markedly improved the memory of the rats as evidenced by the longer duration of the time spent by the rats in the target quadrant looking for the platform, indicating the effectiveness of naringenin in improving the memory of rats. A $100 \mathrm{mg}$ dose of naringenin improved performance more effectively than lower doses of 25 and $50 \mathrm{mg}$.

\section{Discussion}

Isoflurane is a widely used volatile anaesthetic that has been reported to induce neurodegeneration and cognitive impairments in developing brains $(2,5,6,43)$. The hippocampus is the most sensitive region to isoflurane-induced neurotoxicity (52) and earlier studies have demonstrated that neuroapoptosis occurs in the hippocampi following exposure to isoflurane $(8,53)$. In agreement with the findings of previous studies, we observed robust apoptosis in the hippocampi of P7 rats following exposure to isoflurane anaesthesia. It has been demonstrated that the developing brain is particularly vulnerable to anaesthestic-induced neurotoxicity during the period of neurogenesis and synaptogenesis and the $\mathrm{P} 7$ rats have been found to be extremely sensitive to neurotoxic challenge (2). In our study, IHC and western blot analysis revealed enhanced cleaved caspase- 3 expression in the hippocampal tissues of anaesthetic only-exposed rats. Caspase- 3 expression is often used as a potent indicator of apoptosis $(2,6)$ and is also one of 
the final steps in apoptosis (54). Protein expression analysis and IHC data revealed that naringenin effectively inhibited caspase- 3 expression in a dose-dependent manner, suggesting that this downregulation contributed to the decrease in apoptotic cell counts in the hippocampi.

Furthermore, naringenin was also capable of modulating the expression of the $\mathrm{Bcl}-2$ family proteins that regulate mitochondrial membrane integrity and control the release of apoptogenic factors from mitochondria (48). Isoflurane exposure for $6 \mathrm{~h}$ was found to upregulate Bad and Bax proteins and downregulate anti-apoptotic proteins, namely Bcl-xL and Bcl-2. Studies have reported that Bcl-xL enhances cell survival and is widely expressed in the brain. In addition, Bcl-xL maintains mitochondrial membrane integrity and inhibits cytochrome $\mathrm{c}$ release together with Bax $(48,55)$, thus inhibiting apoptosis. Earlier studies of isoflurane have also shown similar results (56). Naringenin upregulated the expression of $\mathrm{Bcl}-\mathrm{xL}$ and Bcl-2 and also caused significant downregulation of Bad and Bax protein expression. These observations indicate that naringenin is effective at inhibiting neurodegeneration.

The results of in vitro experiments have demonstrated the critical role of the PI3K/Akt pathway in neurite initiation, growth and stability (57-59), as well as in regulating the branching of dendrites (60). Propofol exerted neuroprotective effects by activating the PI3/Akt pathway (61). In our study, isoflurane was found to inhibit the phosphorylation of Akt, and to eventually reduce the level of p-GSK-3 $\beta$. Akt plays critical roles in regulating and controlling cell survival and apoptosis. Activation of the PI3K/Akt pathway potentially deactivates the pro-apoptotic factors and activates the anti-apoptotic proteins (62). Isoflurane-induced downregulation of Akt and p-Akt and p-GSK-3 $\beta$ suggests the activation of pro-apoptotic proteins. Activated Akt phosphorylates and inactivates Bad causing the release of the anti-apoptotic protein Bcl-xL that blocks apoptosis by binding to $\mathrm{Bax}(55,63)$. In our study, the administration of naringenin significantly induced the phosphorylation of Akt and GSK-3 $\beta$. Moreover, the isoflurane-induced enhanced expression of PTEN was suppressed by naringenin leading to the activation of the PI3K/Akt signalling cascade. PTEN possesses lipid phosphatase activity and is the negative regulator of the PI3K/Akt pathway (64).

Accumulating evidence has shown that neuro-inflammation is critically involved in anaesthetic-induced neuroapoptosis and cognitive deficits (65-67). Isoflurane induced the release of calcium from the endoplasmic reticulum $(68,69)$, elevated cytosolic calcium levels and this has been suggested to activate the $\mathrm{NF}-\kappa \mathrm{B}$ signalling pathway which is implicated in inflammatory processes $(28,70)$. This activation increased the expression of TNF- $\alpha$, IL-1 $\beta$ and IL-6 (15,66,71). Our results also showed the enhanced expression of NF- $\kappa \mathrm{B}$ p $65, \mathrm{p}-\mathrm{I} \kappa \mathrm{B} \alpha, \mathrm{TNF}-\alpha, \mathrm{IL}-1 \beta$ and IL- 6 suggesting activation of the NF- $\kappa B$ pathway. In addition, the expression of NF- $\kappa \mathrm{B}$ target genes - IAPs - XIAP, cIAP-1 and survivin $(72,73)$ were suppressed by isoflurane, thus enhancing apoptosis. Naringenin, however, modulated the pathway by effectively suppressing TNF- $\alpha$, IL-1 $\beta$ and IL-6 suggesting the involvement of the $\mathrm{NF}-\kappa \mathrm{B}$ signalling pathway in naringenin-mediated neuroprotection.

Cognitive impairments in neonatal rats exposed to isoflurane have been well documented. Moreover, it has been reported that the period of synaptogenesis in the developing brain is highly vulnerable to anaesthetic-induced neurotoxicity (2). Head et al (74) demonstrated that isoflurane causes synaptic injury. In this study, tests for working memory as well as long-term memory were performed to assess whether naringenin was capable of ameliorating isoflurane-induced cognitive deficits. We observed minor alterations in the general behaviour of the animals as indicated in the open field and elevated maze test. However, the spatial working memory was greatly affected following anaesthetic exposure. Isoflurane induced profound alterations in the working memory of the animals as evidenced in the Y-maze test. Working memory is concerned with the cognitive functions that are responsible for synchronized temporary storage and also with the analysis and manipulation of information that is required in order to execute intricate cognitive tasks (75).

Working memory is also involved in higher order cognitive functions such as planning and sequential behaviour. Impairment of the working memory is directly associated with behavioural deficits and involves the hippocampus (76). Thus, isoflurane-induced neurodegeneration in the hippocampi may have directly contributed to the deficits in learning and memory. Notably, we observed a marked improvement in the behaviour and memory of the animals treated with naringenin. The rats exhibited a much improved performance in the Y-maze tests indicating a significant improvement in working memory. A naringenin-induced reduction in hippocampal apoptosis may be in part responsible for the significant improvement. Sanders et al (52) have demonstrated the neuroprotective effects of dexmedetomidine. Dexmedetomidine reduced isoflurane-induced hippocampal neuroapoptosis and also prevented hippocampal-dependent neurocognitive impairment (52).

The MWM test was conducted in order to evaluate the cognition and behaviour of the $\mathrm{P} 35$ rats. It is a reliable test for assessing hippocampal-dependent spatial working and reference memory (51). Jevtovic-Todorovic et al (2) reported that the period of neurogenesis is extremely sensitive to anaesthetic challenge. Active neurogenesis is critical for hippocampal-dependent learning and memory and any negative impact affects the process $(77,78)$. The escape latency time of the rats that received isoflurane alone was significantly longer than the controls. More importantly, neuro-inflammation also mediates isoflurane-induced cognitive impairment $(66,71)$. Nevertheless, we observed a marked improvement in the working memory of the rats treated with naringenin. At all the given doses, naringenin ameliorated memory retention and the cognitive behaviour of the rats. These observations suggest that naringenin may improve long-term memory.

Thus, the marked reduction in neurodegeneration and inhibition of inflammation observed following naringenin administration may have contributed to the improvement in the performance of the rats in the MWM tests. In conclusion, naringenin effectively reduced neuronal apoptosis and neuro-inflammation by modulating the $\mathrm{NF}-\kappa \mathrm{B}$ signalling pathway and also by activateing the PI3K/Akt pathway. Thus, further studies of naringenin are warranted in order to examine the molecular events and targets involved which may aid in developing efficient therapeutic strategies against anaesthetic-induced neuronal toxicity. 


\section{Acknowledgements}

The present study was supported by the fund of the Jiangxi Provincial Department of Science and Technology (no. 20142BBG70067) and the fund of the Jiangxi Provincial Department of Education (no. 81539603).

\section{References}

1. Istaphanous GK and Loepke AW: General anesthetics and the developing brain. Curr Opin Anaesthesiol 22: 368-373, 2009.

2. Jevtovic-Todorovic V, Hartman RE, Izumi Y, Benshoff ND, Dikranian K, Zorumski CF, Olney JW and Wozniak DF: Early exposure to common anesthetic agents causes widespread neurodegeneration in the developing rat brain and persistent learning deficits. J Neurosci 23: 876-882, 2003.

3. Rizzi S, Carter LB, Ori C and Jevtovic-Todorovic V: Clinical anesthesia causes permanent damage to the fetal guinea pig brain. Brain Pathol 18: 198-210, 2008.

4. Satomoto M, Satoh Y, Terui K, Miyao H, Takßishima K, Ito M and Imaki J: Neonatal exposure to sevoflurane induces abnormal social behaviors and deficits in fear conditioning in mice. Anesthesiology 110: 628-637, 2009.

5. Brambrink AM, Evers AS, Avidan MS, Farber NB, Smith DJ, Zhang X, Dissen GA, Creeley CE and Olney JW: Isoflurane-induced neuroapoptosis in the neonatal rhesus macaque brain. Anesthesiology 112: 834-841, 2010.

6. Kong F, Xu L, He D, Zhang X and Lu H: Effects of gestational isoflurane exposure on postnatal memory and learning in rats Eur J Pharmacol 670: 168-174, 2011.

7. Istaphanous GK, Howard J, Nan X, Hughes EA, McCann JC, McAuliffe JJ, Danzer SC and Loepke AW: Comparison of the neuroapoptotic properties of equipotent anesthetic concentrations of desflurane, isoflurane, or sevoflurane in neonatal mice Anesthesiology 114: 578-587, 2011.

8. Liang G, Ward C, Peng J, Zhao Y, Huang B and Wei H: Isoflurane causes greater neurodegeneration than an equivalent exposure of sevoflurane in the developing brain of neonatal mice. Anesthesiology 112: 1325-1334, 2010.

9. Kalkman CJ, Peelen L, Moons KG, Veenhuizen M, Bruens M, Sinnema $G$ and de Jong TP: Behavior and development in children and age at the time of first anesthetic exposure. Anesthesiology 110: 805-812, 2009.

10. Wilder RT, Flick RP, Sprung J, Katusic SK, Barbaresi WJ, Mickelson C, Gleich SJ, Schroeder DR, Weaver AL and Warner DO: Early exposure to anesthesia and learning disabilities in a population-based birth cohort. Anesthesiology 110: 796-804, 2009.

11. Xie Z, Culley DJ, Dong Y, Zhang G, Zhang B, Moir RD, Frosch MP, Crosby G and Tanzi RE: The common inhalation anesthetic isoflurane induces caspase activation and increases amyloid beta-protein level in vivo. Ann Neurol 64: 618-627, 2008

12. Lin D, Cao L, Wang Z, Li J, Washington JM and Zuo Z: Lidocaine attenuates cognitive impairment after isoflurane anesthesia in old rats. Behav Brain Res 228: 319-327, 2012.

13. Zhang Y, Xu Z, Wang H, Dong Y, Shi HN, Culley DJ, Crosby G, Marcantonio ER, Tanzi RE and Xie Z: Anesthetics isoflurane and desflurane differently affect mitochondrial function, learning, and memory. Ann Neurol 71: 687-698, 2012.

14. Shen X, Dong Y, Xu Z, Wang H, Miao C, Soriano SG, Sun D, Baxter MG, Zhang Y and Xie Z: Selective anesthesia-induced neuroinflammation in developing mouse brain and cognitive impairment. Anesthesiology 118: 502-515, 2013.

15. Wu X, Lu Y, Dong Y, Zhang G, Zhang Y, Xu Z, Culley DJ, Crosby G, Marcantonio ER, Tanzi RE and Xie Z: The inhalation anesthetic isoflurane increases levels of proinflammatory TNF- $\alpha$, IL-6, and IL-1ß. Neurobiol Aging 33: 1364-1378, 2012.

16. Wilson CJ, Finch CE and Cohen HJ: Cytokines and cognition - the case for a head-to-toe inflammatory paradigm. J Am Geriatr Soc 50: 2041-2056, 2002.

17. Perry VH: The influence of systemic inflammation on inflammation in the brain: implications for chronic neurodegenerative disease. Brain Behav Immun 18: 407-413, 2004.

18. Goshen I, Kreisel T, Ounallah-Saad H, Renbaum P, Zalzstein Y, Ben-Hur T, Levy-Lahad E and Yirmiya R: A dual role for interleukin-1 in hippocampal-dependent memory processes. Psychoneuroendocrinology 32: 1106-1115, 2007.
19. Rudolph JL, Ramlawi B, Kuchel GA, McElhaney JE, Xie D, Sellke FW, Khabbaz K, Levkoff SE and Marcantonio ER: Chemokines are associated with delirium after cardiac surgery. J Gerontol A Biol Sci Med Sci 63: 184-189, 2008.

20. Teeling JL and Perry VH: Systemic infection and inflammation in acute CNS injury and chronic neurodegeneration: underlying mechanisms. Neuroscience 158: 1062-1073, 2009.

21. Patanella AK, Zinno M, Quaranta D, Nociti V, Frisullo G, Gainotti G, Tonali PA, Batocchi AP and Marra C: Correlations between peripheral blood mononuclear cell production of BDNF, TNF-alpha, IL-6, IL-10 and cognitive performances in multiple sclerosis patients. J Neurosci Res 88: 1106-1112, 2010.

22. Combs CK, Karlo JC, Kao SC and Landreth GE: $\beta$-Amyloid stimulation of microglia and monocytes results in TNFalpha-dependent expression of inducible nitric oxide synthase and neuronal apoptosis. J Neurosci 21: 1179-1188, 2001.

23. Gilmore TD and Wolenski FS: NF-кB: Where did it come from and why? Immunol Rev 246: 14-35, 2012.

24. Zhao Y, Liang G, Chen Q, Joseph DJ, Meng Q, Eckenhoff RG, Eckenhoff MF and Wei $\mathrm{H}$ : Anesthetic-induced neurodegeneration mediated via inositol 1,4,5-trisphosphate receptors. J Pharmacol Exp Ther 333: 14-22, 2010.

25. Zhao YL, Xiang Q, Shi QY, Li SY, Tan L, Wang JT, Jin XG and Luo AL: GABAergic excitotoxicity injury of the immature hippocampal pyramidal neurons' exposure to isoflurane. Anesth Analg 113: 1152-1160, 2011.

26. Zheng $\mathrm{Z}$ and Yenari MA: Post-ischemic inflammation: molecular mechanisms and therapeutic implications. Neurol Res 26: 884-892, 2004

27. Zheng Z, Kim JY, Ma H, Lee JE and Yenari MA: Anti-inflammatory effects of the $70 \mathrm{kDa}$ heat shock protein in experimental stroke. J Cereb Blood Flow Metab 28: 53-63, 2008.

28. Vexler ZS and Yenari MA: Does inflammation after stroke affect the developing brain differently than adult brain? Dev Neurosci 31: 378-393, 2009.

29. Meffert MK, Chang JM, Wiltgen BJ, Fanselow MS and Baltimore D: NF-kappa B functions in synaptic signaling and behavior. Nat Neurosci 6: 1072-1078, 2003.

30. Brunet A, Datta SR and Greenberg ME: Transcription-dependent and-independent control of neuronal survival by the PI3K-Akt signaling pathway. Curr Opin Neurobiol 11: 297-305, 2001.

31. Staal SP: Molecular cloning of the akt oncogene and its human homologues AKT1 and AKT2: Amplification of AKT1 in a primary human gastric adenocarcinoma. Proc Natl Acad Sci USA 84: 5034-5037, 1987.

32. Luo HR, Hattori H, Hossain MA, Hester L, Huang Y, Lee-Kwon W, Donowitz M, Nagata E and Snyder SH: Akt as a mediator of cell death. Proc Natl Acad Sci USA 100: 11712-11717, 2003.

33. Song G, Ouyang G and Bao S: The activation of Akt/PKB signaling pathway and cell survival. J Cell Mol Med 9: 59-71, 2005.

34. Lee HK, Kumar P, Fu Q, Rosen KM and Querfurth HW: The insulin/Akt signaling pathway is targeted by intracellular beta-amyloid. Mol Biol Cell 20: 1533-1544, 2009.

35. Ahmad A, Biersack B,Li Y, Kong D, Bao B, SchobertR, Padhye SB and Sarkar FH: Targeted regulation of PI3K/Akt/mTOR/NF-kB signaling by indole compounds and their derivatives: mechanistic details and biological implications for cancer therapy. Anticancer Agents Med Chem 13: 1002-1013, 2013.

36. Vallverdú-Queralt A, Odriozola-Serrano I, Oms-Oliu G, Lamuela-Raventós RM,Elez-Martínez P and Martín-Belloso O: Changes in the polyphenol profile of tomato juices processed by pulsed electric fields. J Agric Food Chem 60: 9667-9672, 2012.

37. Renugadevi J and Prabu SM: Naringenin protects against cadmium-induced oxidative renal dysfunction in rats. Toxicology 256: 128-134, 2009.

38. Cavia-Saiz M, Busto MD, Pilar-Izquierdo MC, Ortega N, Perez-Mateos M and Muñiz P: Antioxidant properties, radical scavenging activity and biomolecule protection capacity of flavonoid naringenin and its glycoside naringin: a comparative study. J Sci Food Agric 90: 1238-1244, 2010.

39. Ke JY, Kliewer KL, Hamad EM, Cole RM, Powell KA, Andridge RR, Straka SR, Yee LD and Belury MA: The flavonoid, naringenin, decreases adipose tissue mass and attenuates ovariectomy-associated metabolic disturbances in mice. Nutr Metab (Lond) 12: 1, 2015. 
40. Mulvihill EE, Allister EM, Sutherland BG, Telford DE, Sawyez CG, Edwards JY, Markle JM, Hegele RA and Huff MW: Naringenin prevents dyslipidemia, apolipoprotein B overproduction, and hyperinsulinemia in LDL receptor-null mice with diet-induced insulin resistance. Diabetes 58: 2198-2210, 2009.

41. Orliaguet G, Vivien B, Langeron O, Bouhemad B, Coriat P and Riou B: Minimum alveolar concentration of volatile anesthetics in rats during postnatal maturation. Anesthesiology 95: 734-739, 2001.

42. Li Y, Liu C, Zhao Y, Hu K, Zhang J, Zeng M, Luo T, Jiang W and Wang H: Sevoflurane induces short-term changes in proteins in the cerebral cortices of developing rats. Acta Anaesthesiol Scand 57: 380-390, 2013a.

43. Li Y, Wang F, Liu C, Zeng M, Han X, Luo T, Jiang W, Xu J and Wang H: JNK pathway may be involved in isoflurane-induced apoptosis in the hippocampi of neonatal rats. Neurosci Lett 545 17-22, 2013b

44. Li Y, Liang G, Wang S, Meng Q, Wang Q and Wei H: Effects of fetal exposure to isoflurane on postnatal memory and learning in rats. Neuropharmacology 53: 942-950, 2007.

45. Satoh Y, Endo S, Ikeda T, Yamada K, Ito M, Kuroki M, Hiramoto T, Imamura O, Kobayashi Y, Watanabe Y, et al: Extracellular signal-regulated kinase 2 (ERK2) knockdown mice show deficits in long-term memory; ERK2 has a specific function in learning and memory. J Neurosci 27: 10765-10776, 2007.

46. Kodama M, Satoh Y, Otsubo Y, Araki Y, Yonamine R, Masui K and Kazama T: Neonatal desflurane exposure induces more robust neuroapoptosis than do isoflurane and sevoflurane and impairs working memory. Anesthesiology 115: 979-991, 2011.

47. Wei H, Kang B, Wei W, Liang G, Meng QC, Li Y and Eckenhoff RG: Isoflurane and sevoflurane affect cell survival and BCL-2/BAX ratio differently. Brain Res 1037: 139-147, 2005

48. Zhao H, Yenari MA, Cheng D, Sapolsky RM and Steinberg GK: $\mathrm{Bcl}-2$ overexpression protects against neuron loss within the ischemic margin following experimental stroke and inhibits cytochrome $c$ translocation and caspase-3 activity. J Neurochem 85: 1026-1036, 2003.

49. Jones MW: A comparative review of rodent prefrontal cortex and working memory. Curr Mol Med 2: 639-647, 2002.

50. Saxe MD, Battaglia F, Wang JW, Malleret G, David DJ, Monckton JE, Garcia AD, Sofroniew MV, Kandel ER, Santarelli L, et al: Ablation of hippocampal neurogenesis impairs contextual fear conditioning and synaptic plasticity in the dentate gyrus. Proc Natl Acad Sci USA 103: 17501-17506, 2006.

51. D'Hooge R and De Deyn PP: Applications of the Morris water maze in the study of learning and memory. Brain Res Brain Res Rev 36: 60-90, 2001.

52. Sanders RD, Xu J, Shu Y, Januszewski A, Halder S, Fidalgo A, Sun P, Hossain M, Ma D and Maze M: Dexmedetomidine attenuates isoflurane-induced neurocognitive impairment in neonatal rats. Anesthesiology 110: 1077-1085, 2009.

53. Li Y, Zeng M, Chen W, Liu C, Wang F, Han X, Zuo Z and Peng S: Dexmedetomidine reduces isoflurane-induced neuroapoptosis partly by preserving PI3K/Akt pathway in the hippocampus of neonatal rats. PLoS One 9: e93639, 2014.

54. Thornberry NA and Lazebnik Y: Caspases: enemies within. Science 281: 1312-1316, 1998.

55. Hsu SY, Kaipia A, Zhu L and Hsueh AJ: Interference of BAD (Bcl-xL/Bcl-2-associated death promoter)-induced apoptosis in mammalian cells by 14-3-3 isoforms and P11. Mol Endocrinol 11: $1858-1867,1997$.

56. Yon JH, Daniel-Johnson J, Carter LB and Jevtovic-Todorovic V: Anesthesia induces neuronal cell death in the developing rat brain via the intrinsic and extrinsic apoptotic pathways. Neuroscience 135: 815-827, 2005.

57. Atwal JK, Massie B, Miller FD and Kaplan DR: The TrkB-Shc site signals neuronal survival and local axon growth via MEK and P13-kinase. Neuron 27: 265-277, 2000.

58. Sanchez S, Sayas CL, Lim F, Diaz-Nido J, Avila J and Wandosell F: The inhibition of phosphatidylinositol-3-kinase induces neurite retraction and activates GSK3. J Neurochem 78: 468-481, 2001.
59. Dijkhuizen PA and Ghosh A: BDNF regulates primary dendrite formation in cortical neurons via the PI3-kinase and MAP kinase signaling pathways. J Neurobiol 62: 278-288, 2005.

60. Jaworski J, Spangler S, Seeburg DP, Hoogenraad CC and Sheng M: Control of dendritic arborization by the phosphoinositide-3'-kinase-Akt-mammalian target of rapamycin pathway. J Neurosci 25: 11300-11312, 2005.

61. Wang HY, Wang GL, Yu YH and Wang Y: The role of phosphoinositide-3-kinase/Akt pathway in propofol-induced postconditioning against focal cerebral ischemia-reperfusion injury in rats. Brain Res 1297: 177-184, 2009.

62. Liu P, Cheng H, Roberts TM and Zhao JJ: Targeting the phosphoinositide 3-kinase pathway in cancer. Nat Rev Drug Discov 8: 627-644, 2009

63. Koh PO: Nicotinamide attenuates the ischemic brain injury-induced decrease of Akt activation and Bad phosphorylation. Neurosci Lett 498: 105-109, 2011.

64. Zhang J, Yu XH, Yan YG, Wang C and Wang WJ: PI3K/Akt signaling in osteosarcoma. Clin Chim Acta 444: 182-192, 2015.

65. Li ZQ, Rong XY, Liu YJ, Ni C, Tian XS, Mo N, Chui DH and Guo XY: Activation of the canonical nuclear factor- $\kappa B$ pathway is involved in isoflurane-induced hippocampal interleukin-1 $\beta$ elevation and the resultant cognitive deficits in aged rats. Biochem Biophys Res Commun 438: 628-634, 2013.

66. Cao L, Li L, Lin D and Zuo Z: Isoflurane induces learning impairment that is mediated by interleukin learning impairment in rodents. PLoS One 7: e51431, 2012.

67. Shu Y, Zhou Z, Wan Y, Sanders RD, Li M, Pac-Soo CK, Maze M and Ma D: Nociceptive stimuli enhance anesthetic-induced neuroapoptosis in the rat developing brain. Neurobiol Dis 45: 743-750, 2012.

68. Liang G, Wang Q, Li Y, Kang B, Eckenhoff MF, Eckenhoff RG and Wei $\mathrm{H}$ : A presenilin-1 mutation renders neurons vulnerable to isoflurane toxicity. Anesth Analg 106: 492-500, 2008.

69. Yang H, Liang G, Hawkins BJ, Madesh M, Pierwola A and Wei $\mathrm{H}$ : Inhalational anesthetics induce cell damage by disruption of intracellular calcium homeostasis with different potencies. Anesthesiology 109: 243-250, 2008.

70. Kim YJ, Hwang SY, Oh ES, Oh S and Han IO: IL-1beta, an immediate early protein secreted by activated microglia, induces iNOS/NO in C6 astrocytoma cells through p38 MAPK and NF-kappaB pathways. J Neurosci Res 84: 1037-1046, 2006.

71. Lin D and Zuo Z: Isoflurane induces hippocampal cell injury and cognitive impairments in adult rats. Neuropharmacology 61: 1354-1359, 2011.

72. Hunter AM, LaCasse EC and Korneluk RG: The inhibitors of apoptosis (IAPs) as cancer targets. Apoptosis 12: 1543-1568, 2007.

73. Gyrd-Hansen M and Meier P: IAPs: From caspase inhibitors to modulators of NF-kappaB, inflammation and cancer. Nat Rev Cancer 10: 561-574, 2010.

74. Head BP, Patel HH, Niesman IR, Drummond JC, Roth DM and Patel PM: Inhibition of 75 neurotrophin receptor attenuates isoflurane-mediated neuronal apoptosis in the neonatal central nervous system. Anesthesiology 110: 813-825, 2009.

75. Baddeley A: Working memory. Science 255: 556-559, 1992.

76. Morris RG, Garrud P, Rawlins JN and O'Keefe J: Place navigation impaired in rats with hippocampal lesions. Nature 297: 681-683, 1982

77. Madsen TM, Kristjansen PE, Bolwig TG and Wörtwein G: Arrested neuronal proliferation and impaired hippocampal function following fractionated brain irradiation in the adult rat. Neuroscience 119: 635-642, 2003.

78. Rola R, Raber J, Rizk A, Otsuka S, VandenBerg SR, Morhardt DR and Fike JR: Radiation-induced impairment of hippocampal neurogenesis is associated with cognitive deficits in young mice. Exp Neurol 188: 316-330, 2004. 